\title{
Estimation of the driven gas turbine unit technical performance using the standard measuring systems
}

\author{
Vitalii L. Blinov ${ }^{1, *}$, Oleg V. Komarov ${ }^{1}$, and Egor A. Zaslavskiy ${ }^{1}$ \\ ${ }^{1}$ Ural Federal University, Turbines and Engines Department, 620002 Yekaterinburg, Russia
}

\begin{abstract}
In large pipeline gas transport systems the operation and maintenance of gas pumping units are carried according to the current number of equivalent working hours of centrifugal gas compressors and gas turbines. Modern terms of lean production require the maintenance procedure to be done according to the current technical performance of equipment. The paper presents a designed and verified methodology of technical performance estimation of gas turbine units using the standard measuring systems. This method includes a verified high-order mathematical model based on the gas dynamic function for the precise analytical description of turbomachinery aerodynamics. The models are defined for different types of multishaft gas turbines. In this article the results of technical performance estimation of different gas turbine units are discussed.
\end{abstract}

\section{Introduction}

In large pipeline gas transport systems the operation and maintenance of gas pumping units are carried out according to the current number of equivalent working hours of centrifugal gas compressors and gas turbine units (GTU). Modern terms of lean production require the maintenance procedure to be done according to the current technical performance of equipment. The estimation of the current technical performance of GTU is essential for its proper utilization, maintenance and extension of its operation time between failures and planned overhauls [1,2].

To date, in the gas transportation systems few algorithms addressed to online control of equipment efficiency and degradation coefficient during its life time are used. The basic explanation of the degradation reasons has been extensively studied and presented in [35] as an example. It is worth noting that the accurate determination of the gas turbines (GT) current power is a key point.

One of the most widespread solutions for power consumption definition is the introduction of a torque meter in the shaft between a power turbine and a gas compressor [6]. However, in the current operating conditions, this method is extremely difficult since most units are not equipped with a torque meter. Another solution is the estimation of the power consumed by a gas compressor [5,7]. This method requires the proper measurement of the natural gas massflow which is not always possible. Gas path analysis methods for gas turbine performance estimation are also used [8,9]. Special attention is paid to the gas turbines with the flow part variable geometry of the power turbine (PT) [10].
In any case, a gas pumping station remains a very complicated system to have enough measuring facilities and human power for estimation of the real performance of every module, so mathematical models still has opportunities for implementation [2,9].

\section{Materials and methods}

Most of the GTU for the mechanical drive have two or three shaft designs [2]. Aero derivative, marine and stationary drives are used [11]. For every type of the gas turbine a similar approach is applied to adopt a system of parametrical diagnostics by thermal and aerodynamic parameters. The key feature of this approach is that only standard measured parameters are considered for a gas turbine unit. No additional power measurements, like a torque meter or measurement of the power consumed by a gas compressor, are involved.

The proposed approach can be divided into three stages (Figure 1). At the first stage, a mathematical model of gas turbines (according to nominal load mode parameters) is developed. At the second stage, it is checked by using test results. Having verified the data, the approach can be used to assess the technical condition of gas turbines only if standard measured parameters are used. This is the third stage.

\subsection{Mathematical model of GTU}

The following parameters are used as input data:

- Ambient pressure and temperature.

- GTUs nominal parameters taken according to the GT catalogue issued by VNIIGAZ [11]: nominal power output, efficiency of GT, fuel gas consumption, cycle

Corresponding author: v.l.blinov@ urfu.ru 


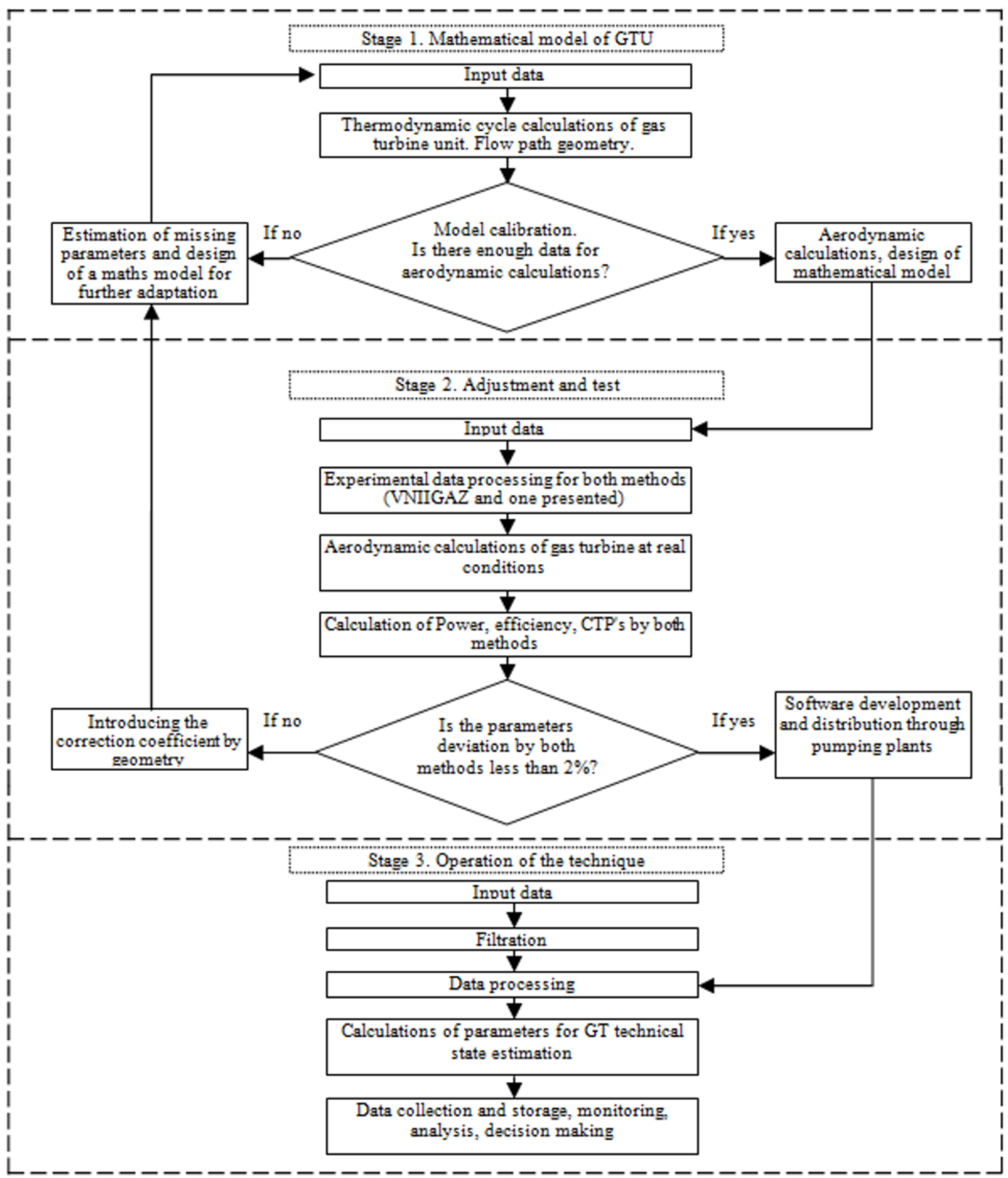

Fig. 1. Architecture of the driven gas turbine unit technical performance estimation with standard measurements.

airflow, mass flow of working gas in turbine after PT, air compressor pressure ratio, temperature after compressor, turbine inlet temperature, temperature of gases before or after PT, hydraulic pressure drop in inlet and outlet ducts, shafts speed. It should be noted that this catalogue [11] is not a producer statement declaration about any GT parameter, but really measured data after the acceptance testing of each unit on the site and further statistical averaging.

- Variable parameters: mechanical losses; efficiency of every turbine, combustor, and axial compressor; the speed coefficient in stator and rotor blades. Also the relative coefficient is used to consider the difference in the airflow through a turbine and a compressor. These parameters must be verified and adjusted within the adjustment and test stage.

- Geometry of the real flow path of turbines: diameters and height of blades.

The thermodynamic calculations were made according to [12]. At this stage the main parameters are: specific heat, temperature and pressure after compressor, specific compression work; then pressure and 

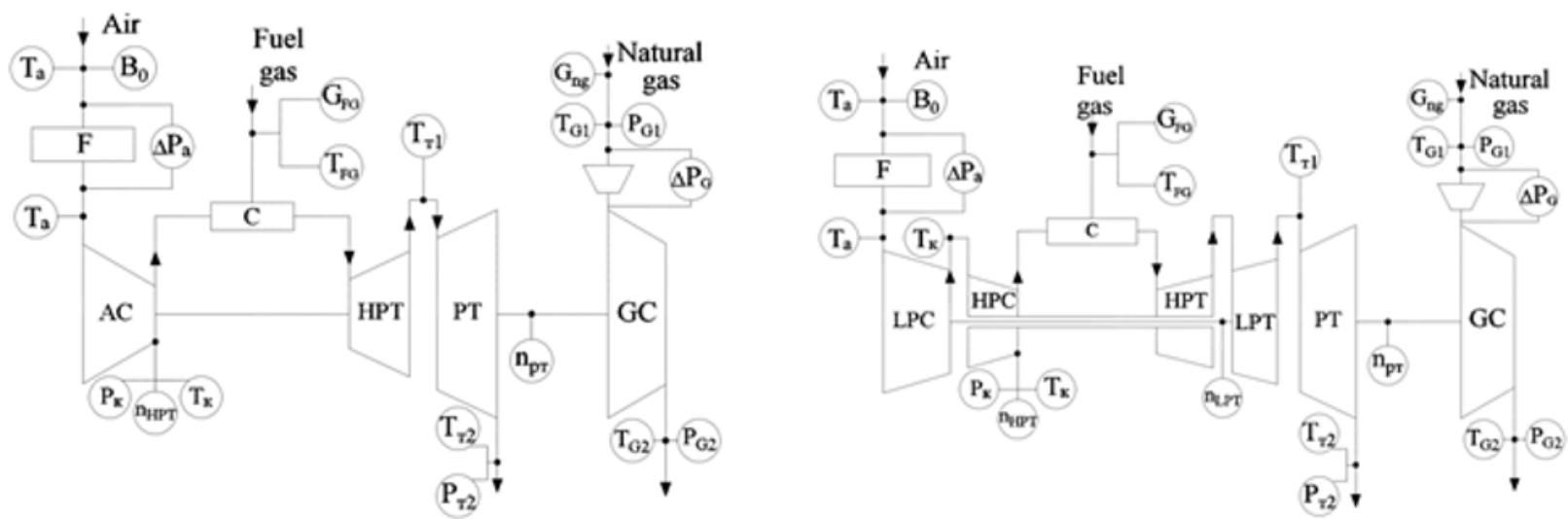

Fig. 2. Principal scheme for two (left) and three (right) shaft GTUs: F - filter; AC - axial compressor; LPC/HPC - low/high pressure compressor; $\mathrm{C}$ - combustor; HPT/LPT - high/low pressure turbine; PT - power turbine; GC - natural gas compressor.

temperature at turbines inlet and outlet, pressure ration distribution between turbines, airflow, specific expansion work, etc. Additionally, the basic geometric parameters of the turbines flow part are determined at this stage.

The next step is model calibration. The aim of this model calibration is to achieve good convergence between the computed integral parameters of GT at nominal mode and ones from the catalogue [11]. If necessary, some adjustable parameters can be varied, such as efficiency of modules and turbine/compressor mass flow.

The aerodynamic calculations of turbine stages are done according to the methodology described in [12] which consists of the basic algorithm for velocity diagrams correlations together with reaction, load coefficients and basic thermodynamic. As a result, pressures and temperature (static and total) at all stages together with kinematic parameters are calculated with the use of real flow path geometry.

\subsection{Adjustment and test}

The input data for this stage is the measured parameters of the gas turbine during testing. The principal schemes for two and three shaft GTUs are presented in Figure 2.

Also the input data is basic and adjustable parameters such as: swept areas and flow angles at each stator stage outlet, kinematic reaction at mid span, etc. (taken from nominal mode calculations at the previous stage).

Processing of the test results is carried out by two methods: VNIIGAZ [7] and one presented. In the first case, the effective power and efficiency of a gas turbine are determined by the parameters of a natural gas compressor. The gas flow was determined by an ultrasonic flow meter.

In the second case, the PT real effective work and gas mass flow are used to calculate the effective power of the GTU. The experimental data processing differs from the thermodynamic cycle calculations of GTU in the nominal mode using the measured inlet and outlet temperatures and pressures of the axial compressor and power turbine for calculating. This allows us to determine with sufficient accuracy the thermodynamic parameters of each turbine stage and the specific effective work of the PT, $\mathrm{kJ} / \mathrm{kg}$ :

$$
H_{e}=C_{p} \cdot T_{t 1} \cdot\left(1-\left(\frac{P_{t 1}}{P_{t 2}}\right)^{\frac{1-k}{k}}\right) \cdot \frac{G_{t}}{G_{a}} \cdot \eta_{\mathrm{t}} \cdot \eta_{\text {mech }}
$$

where $\mathrm{C}_{\mathrm{p}}$ - gas heat capacity at constant pressure, $\mathrm{kJ} / \mathrm{kg} / \mathrm{K} ; \mathrm{T}_{\mathrm{t} 1}-\mathrm{PT}$ inlet temperature, $\mathrm{K} ; \mathrm{P}_{\mathrm{t} 1}, \mathrm{P}_{\mathrm{t} 2}-\mathrm{PT}$ pressure inlet and outlet respectively, $\mathrm{Pa} ; \mathrm{k}$ - ratio of specific heats under total temperature; $\mathrm{G}_{\mathrm{t}}-\mathrm{PT}$ mass flow at the nominal operating mode of GTU, $\mathrm{kg} / \mathrm{s} ; \mathrm{G}_{\mathrm{a}}-$ air consumption in GTU at the nominal operating mode, $\mathrm{kg} / \mathrm{s} ; \eta_{\mathrm{t}}$ - adiabatic turbine efficiency; $\eta_{\text {mech }}-$ mechanical turbine efficiency.

Parameter $\mathrm{G}_{\mathrm{t}} / \mathrm{G}_{\mathrm{a}}$ as well as mechanical and adiabatic turbine efficiency is accepted according to the results of the GTU model calculation at the previous stage. If the blades geometry of a turbine without cooling is known, then the curve of the efficiency can be estimated using the results of CFD analysis. Otherwise, the efficiency value can be taken from the known range [2,12]. For turbines with cooling the efficiency can be estimated using the known methods [13].

The next step is aerodynamic calculations of a gas turbine at real conditions. The purpose of this step is to determine the parameters for the PT mass flow calculation in the current operation mode (a test mode). The mass flow of working gases through PT for the power output calculation $\mathrm{N}_{\mathrm{e}}$ calc is based on gas dynamic functions (GDF) [14] by the following equation:

$$
G=\frac{m \cdot P^{*} \cdot q(\lambda) \cdot F}{\sqrt{T^{*}}},
$$

where $\mathrm{F}$ - swept area in considered section, $\mathrm{m}^{2} ; \mathrm{T}^{*}-$ total temperature in considered section, $\mathrm{K} ; \mathrm{P}^{*}-$ total pressure in considered section, $\mathrm{H} / \mathrm{m}^{2} ; \mathrm{q}(\lambda)-$ GDF of reduced mass flow density; $\mathrm{m}$ - constant coefficient of gas:

$$
m=\sqrt{\left(\frac{2}{k+1}\right)^{\frac{k+1}{k-1}} \cdot \frac{k}{R}}
$$


where $\mathrm{R}$ - universal gas constant, $\mathrm{m}^{2} / \mathrm{s}^{2} \mathrm{~K}$.

Total pressure in considered section is:

$$
P^{*}=\frac{P \cdot C_{a}^{2}}{2 R \cdot T \cdot j^{*}(\lambda)}
$$

where $\mathrm{j}^{*}(\lambda)$ - GDF of dynamic pressure; P and T - static pressure and temperature in considered section that are determined in the previous step (experimental data processing); $\mathrm{C}_{\mathrm{a}}-$ axial component of flow gas velocity in section, $\mathrm{m} / \mathrm{s}$ :

$$
C_{a}=\phi \cdot \sqrt{2 \cdot(1-\rho) \cdot \frac{H}{\eta}} \cdot \sin \alpha,
$$

where $\phi-$ speed coefficient in stator or rotor blades (in considered section); $\rho$ - kinematic reaction at mid span; $\mathrm{H}$ - specific expansion work of considered turbine stage; $\eta$ - adiabatic efficiency of turbine stage; $\alpha$ - outlet flow angle in considered section. These parameters are determined at the stages «Mathematical model of GTU» and «Experimental data processing».

The GDF of dynamic pressure is:

$$
\begin{gathered}
j^{*}(\lambda)=\frac{k}{k+1} \cdot \lambda^{2} \cdot\left(1-\frac{k-1}{k+1} \cdot \lambda^{2}\right)^{\frac{1}{k-1}}, \\
\lambda=\frac{C_{a}}{a_{c r}} \\
a_{c r}=\sqrt{2 \cdot(k / k+1) \cdot R \cdot T^{*}}, \\
T^{*}=T+\frac{C_{a}^{2}}{2 \cdot C_{p}},
\end{gathered}
$$

where $\lambda$ - reduced velocity; $\mathrm{a}_{\mathrm{cr}}$ - speed of gas in critical throat, $\mathrm{m} / \mathrm{s}$.

The gas dynamic function of reduced mass flow density is determined from:

$$
q(\lambda)=\left(\frac{k+1}{2}\right)^{\frac{1}{k-1}} \cdot \lambda \cdot\left(1-\frac{k-1}{k+1} \cdot \lambda^{2}\right)^{\frac{1}{k-1}},
$$

The PT specific expansion work (1) and mass flow (2) are defined according to this model at the experimental data processing stage and then the power output can be obtained.

The final iteration of calculations takes place for the following parameters: corrected power output $\mathrm{N}_{\mathrm{e}}$ cor, corrected PT shaft speed $\mathrm{n}_{\mathrm{PT}}$ cor, corrected massflow through $\mathrm{PT} \mathrm{G}_{\mathrm{FG}}$ cor, efficiency $\eta_{\mathrm{e}}$ cor for every recorded operating mode. The turbine parameters with nominal corrected outlet temperature $\mathrm{T}_{\mathrm{t} 2 \text { cor }}{ }^{\text {nom }}$ for these modes are defined.

The power at the nominal PT outlet temperature $\mathrm{T}_{\mathrm{t} 2}$ cor ${ }^{\text {nom }}$ can be defined according to Figure 3 where it is graphically presented for one of the GTUs.

The final stage of the procedure is the calculation of GTU coefficients of the technical performance (CTP). CTP can be determined from the ratio of real power, efficiency or fuel gas consumption at the nominal operating mode to the passport (ideal) data of the gas turbine [15]. CTP calculated by power is used more often and is defined by the following equation:

$$
K_{N e}=\frac{N_{e c o r}^{n o m}}{N_{e}^{n o m}},
$$

$\mathrm{N}_{\mathrm{e}}$ cor ${ }^{\text {nom }}$ and $\mathrm{Ne}_{\mathrm{e}}{ }^{\text {nom }}$ - power of GT, corrected to nominal outlet temperature $\mathrm{T}_{\mathrm{t} 2}$ cor ${ }^{\text {nom }}$, and ideal (based on producer's documentation) power of GT.

At the next step the comparison takes place between the results obtained within the presented methodology and the VNIIGAZ methodology [7].

In some cases the difference between these two values exceeded $2 \%$. This is due to the fact that a load factor and other stage parameters at particular GT type might not be chosen in the optimal range. And the behavior of this design differs from the expected one.

To adjust this universal model to the exact geometry reality corrective coefficient is introduced (called constriction coefficient) in the calculation of massflow through PT. So the mass flow is powered by this coefficient and the linear dependency is observed.

\subsection{Operation of the technique}

Based on the presented methodology, the in-house code can be developed and distributed among gas pumping plants for statistical information collection. Input data is standard measured parameters of gas turbines.

It is essential to filter this data before use. First of all, the start and stop modes of gas turbines should be excluded from the analysis. It is also necessary to identify transient operating modes of gas turbines, when the gas turbine operation parameters change significantly in a short period of time. For example, this happens when the load rises or falls, or when the outside temperature changes significantly. The filtration algorithm should track the change in the main parameters of the gas turbine operation (the shafts speed and the temperature of gases in different sections). If the change in parameters exceeds a predetermined value within a given time range, then this time interval is excluded from the analysis. It is also necessary to exclude the operating time of the gas turbine when there is no measurement of any parameter or the diagnostic system has identified a malfunction of the measuring equipment.

Then, calculations are carried out according to the presented approach and the current gas turbine power values in different modes are determined. After that, it is necessary to correct all the parameters to the nominal conditions. Using this data, the graphs of GTU power changes are built (Figure 3). Gas turbine power trends are linear. All "dropped points" are excluded from the further analysis and the power trend is being rebuilt. Such points are determined by the standard deviation of the current power value from the GT power trend. Then the available power, efficiency and CTP of the gas turbine (11) are determined using these trends. 
Table 1. Information about the GTUs.

\begin{tabular}{|c|c|c|c|c|c|}
\hline Designation & Type & Cycle & Shaft & Rated power & Efficiency \\
\hline GTU-10 & stationary & regenerative & 2 & 10,0 & 29,0 \\
\hline GTU-16 & aero derivative & simple & 3 & 16,0 & 27,5 \\
\hline GTU-25 & aero derivative & simple & 3 & 25,0 & 38,7 \\
\hline GTU-30 & stationary & simple & 2 & 30,7 & 36,0 \\
\hline
\end{tabular}

Monitoring these results makes it possible to track the performance degradation of gas turbines. The data can be used for prediction and decision-making on maintenance and repair of gas turbine units $[16,17]$.

\section{Results}

The described technique was tested on different types of gas turbine units in the gas transport system. A comparison of the results for the two approaches was carried out. Four types of gas turbines of different capacities $(10,16,25$, and $30 \mathrm{MW})$ were tested and information about the GTUs is presented in the table [11].

Measurement schemes are presented in Figure 2.

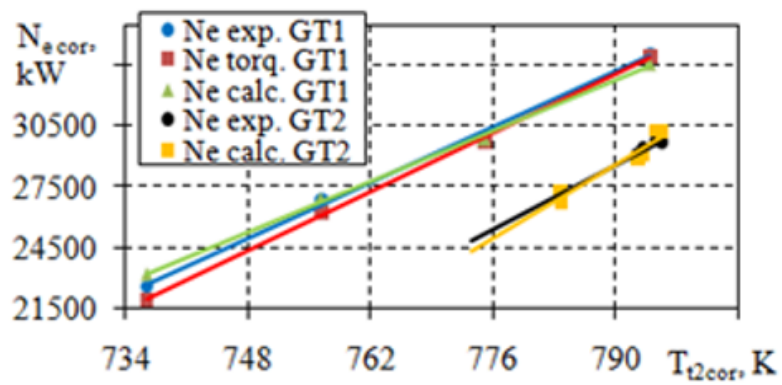

Fig. 3. Test data for GTU-30.

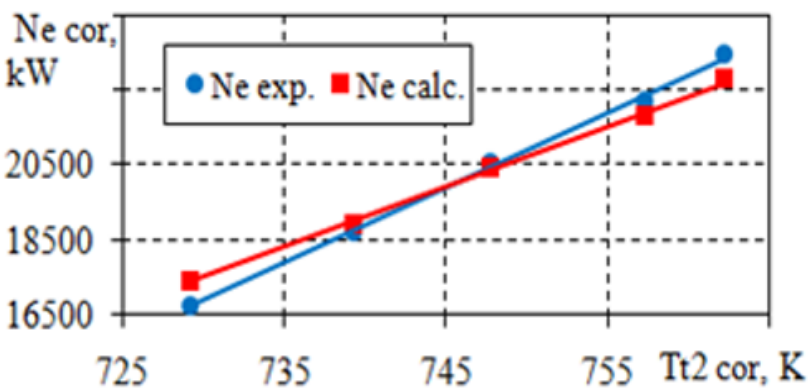

Fig. 4. Test data for GTU-25 (left) and GTU-16 (right).

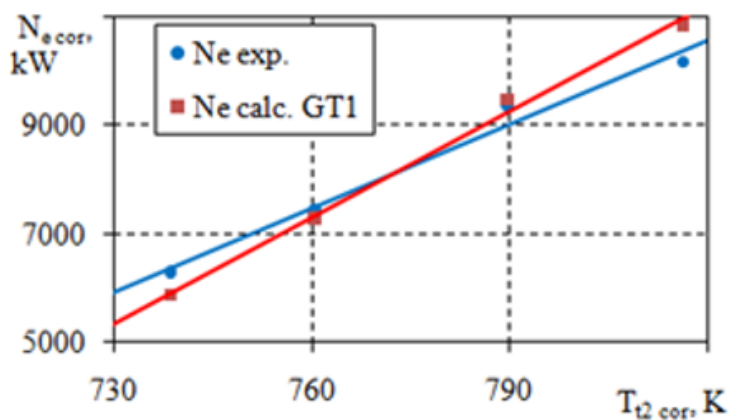

Fig. 5. Test data for GTU-10.
After the experimental data processing, the output power was determined by two methods: VNIIGAZ $\left(\mathrm{N}_{\mathrm{e}}\right.$ exp. $)$ and the presented methodology $\left(\mathrm{N}_{\mathrm{e}}\right.$ calc.). It should be noted that the accuracy of the test results significantly depends on the quality of the measurements and the accuracy of the measuring instruments [18], that is why the measuring equipment was calibrated to meet requirements of GOST standard [19]. The results of determining the power are presented in Figures 3-5.

\section{Discussion}

The experimental data for two GTUs-30 is presented in Figure 3. The trend line is developed with the use of the measured points and then extrapolated down to the
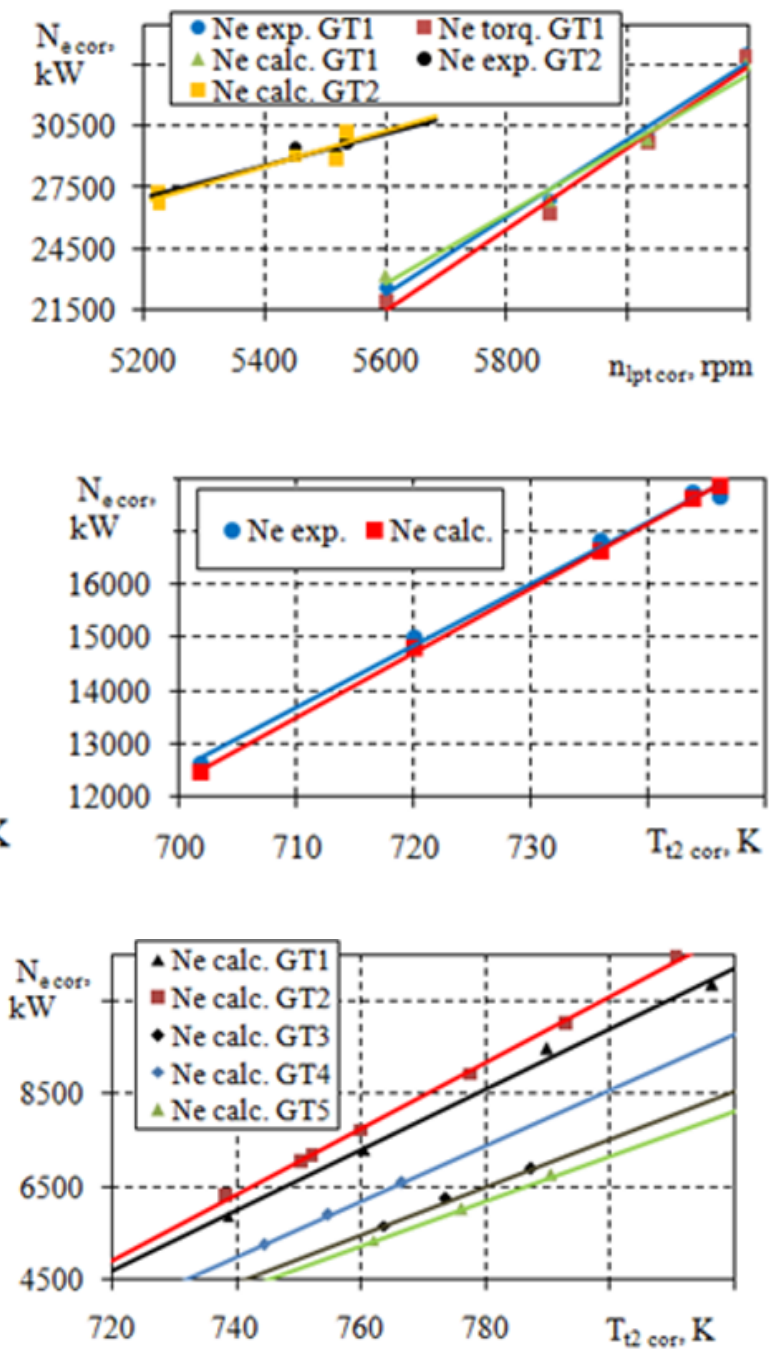
nominal exhaust temperature (figure 3, left) and the nominal shaft speed (figure 3, right), where the expected power output is estimated and then compared with nominal one. The GT1 was tested in winter and the GT2 - in summer. It is known that in summer gas turbine power limitation occurs due to reaching the maximum exhaust temperature of gases. In winter, one occurs when the maximum shafts speed is reached. The CTP of a gas turbine must be determined by the parameter that reaches the maximum value under the current operating conditions before others [15]. If one determines the CTP of GT1 by $\mathrm{T}_{\mathrm{t} 2 \mathrm{cor}}$ and CTP of GT2 by $\mathrm{n}_{1 \mathrm{pt} \text { cor. }}$ then the CTP of both units will be about 1,0. However, in fact, the

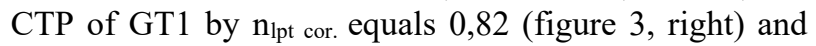
the CTP of GT2 by $\mathrm{T}_{\mathrm{t} 2 \text { cor. }}$ equals 0,88 (figure 3 , left). It should be noticed that the line of GT2 extrapolated from a narrow set of points so there might lead to a mistake in the inclination angle of this line. Nevertheless, it can be seen that these points are significantly distant from the line of GT1.

The power values determined by both methods coincide with an accuracy of $2 \%$ for both gas turbines. For GT1, the power was also determined using a torque meter $\left(\mathrm{N}_{\text {etorq. }}\right)$. The standard deviation of $\mathrm{N}_{\text {etorq. }}$ from the power determined by thermodynamic parameters does not exceed $2,5 \%$. The deviation of gas turbine power determined by several approaches can be used as a criterion for the reliability of experimental data in gas turbine diagnostic systems, as well as for calibrating one approach relative to another.

Figure 4 shows the experimental data for GTU-25 (left) and GTU-16 (right). The standard deviation from the VNIIGAZ methodology was 2,1 and 1,5\% for GTU25 and GTK-16 respectively. Figure 5 represents the experimental data for several GTUs-10. The standard deviations from the VNIIGAZ methodology for different gas turbines were in the range of $1-5 \%$.

The statistical data for five units is presented in Figure 5 (right). All points here are calculated according to the presented methodology by using only standard measurements with recalibrated measuring equipment. The lines here represent the trend of corresponding sets of points. It is clear that these units have different values of CTP. This is mainly due to the fact that the units have different operating hours after repair. Nominal exhaust temperature for GTU-10 is $780 \mathrm{~K}$. All five trends crossing nominal temperature at power turbine outlet (corrected) in different locations are: 9250, 8620, 7400, 6510 , and $6250 \mathrm{~kW}$ respectively. So GT№1 has 0,925 , №2 - 0,862, №3 - 0,740, №4 - 0,651, and №5 - 0,625. It can be seen that GT № 3, 4 and 5 have an unsatisfactory technical condition. It is worth noting that the units have a total operating time of more than 130 000 hours, and in some cases the operating time reaches 200000 hours. Today these type units are being replaced with the new ones.

The degradation coefficient of gas turbines can be estimated by the shift of the trend line. For this, the exact value of the power of a gas turbine is not necessary to determine and it is enough to track the change in power (for example, in \%). Also, to start tracking is not necessary with the "ideal" state of the gas turbine. This feature may be used in diagnostic systems.

The presented method can also be used for quantitative estimation of maintenance procedures at GTU based only on standard measurements. Any unit can be tested before and after washing [20] or tip clearance recovery in an axial compressor, and, as a result, CTP gain can be defined. The same test series were carried out on marine derivative GTU before and after compressor washing [9].

\section{Conclusions}

The method of power output estimation is realized and showed its good convergence with the method based on the power consumption of a gas compressor. The method accuracy is within the measurement error. The use of standard measurement systems in most cases is sufficient to determine the CTP of GTU. The measurement accuracy in most cases complies with GOST [19]. The presented methodology has several adjustable parameters for gas dynamic functions that are to be defined and properly verified based on the first set of special experiments and technical documentation. After its validation, the model can be implemented for all machines of this type in real time.

\section{References}

[1] P.P. Walsh, P. Fletcher, Gas turbine performance (Blackwell Science Ltd a Blackwell Publishing company, 2004)

[2] B.S. Revzin, Gas-pumping units with a gasturbine drive (transl.) (UGTU-UPI, Ekaterinburg, 2002)

[3] R. Kurz, K. Brun, Degradation in gas turbine systems, Journal of Engineering for Gas Turbines and Power 123, 70-77 (2001)

[4] D. Burnes, R. Kurz, Performance Degradation Effects in Modern Industrial Gas Turbines, Proceedings of Zurich Global Power and Propulsion Forum (2018)

[5] A.P. Tarabrin, V.A. Schurovsky, A.I. Bodrov, J.P. Stalder, Influence of axial compressor fouling on gas turbine unit performance based on different schemes and with different initial parameters, ASME Paper 98-GT-416 (1998)

[6] R.D. van Millingen, J.D. van Millingenp, Phase Shift Torquemeters for Gas Turbine Development and Monitoring, International Gas Turbine and Aeroengine Congress and Exposition, Orlando (1991)

[7] V.A. Shurovskiy, Yu.N. Sinicyn, V.I. Korneev, A.V. Cheremin, G.S. Stepanov, Guidelines for conducting thermal engineering and gas-dynamic calculations during testing of gas turbine gas pumping units (transl.) (VNIIGAZ, 1999)

[8] Y.G. Li, Gas Turbine Performance and Health Status Estimation Using Adaptive Gas Path 
Analysis, Article in Journal of Engineering for Gas Turbines and Power 132, 4 (April 2010)

[9] O.V. Komarov, V.L. Blinov, V.A. Sedunin, A.V. Skorochodov, Parametrical Diagnostics of Gas Turbine Performance On Side At Gas Pumping Plants Based On Standard Measurements, Proceedings of ASME Turbo Expo, GT2014$25392(2014)$

[10] O.V. Komarov, V.L. Blinov, V.A. Sedunin, On Technical Performance Estimation of a Gas Turbine with Variable Power Turbine Vanes, Procedia Engineering 150, 2nd International Conference on Industrial Engineering, 1378-1383 (2016)

[11] Album of parameters for gas turbine gas pumping units (transl.) (VNIIGAZ, 2006)

[12] O.V. Komarov, V.L. Blinov, A.S. Shemyakinsky, Thermal and gas-dynamic calculations of gas turbine units: a training manual (transl.) (Ural Federal University, Ekaterinburg, 2018)

[13] J.H. Horlock, L. Torbidoni, Calculations of Cooled Turbine Efficiency, Journal of Engineering for Gas Turbines and Power 130, 1 (January 2008)

[14] A.A. Dimentova, F.S. Reksitin, V.A. Ryabov, Tables of gas-dynamic functions (transl.) (Mashinostroenie, Moscow, 1966)

[15] A.V. Semushkin, A.O. Podlozny, Ye.A. Chernikova, V.A. Shchurovskiy, Methodical grounds for parametric diagnostics of turbine gascompressor units, News of gas science 1, 29, 2231 (2017)

[16] I.B. Murmanskii, K.E. Aronson, V.L. Blinov, N.V. Zhelonkin and B.E. Murmansky, Digital diagnostic complex for power turbine units equipment, IOP Conf. Series: Materials Science and Engineering 643, 012109 (2019)

[17] S. Ahsan, T.A. Lemma,. Remaining Useful Life Prediction of Gas Turbine Engine using Autoregressive Model, MATEC Web of Conferences 131, 04014 (2017)

[18] M. Pinelli, P.R. Spina, Gas Turbine Field Performance Determination: Sources of Uncertainties, Article in Journal of Engineering for Gas Turbines and Power 124, 1 (January 2002)

[19] Gas turbines, Test methods. Acceptance tests, ISO/DIS 2314 (2007)

[20] K.K. Botros, H. Golshan, D. Rogers, Effects of Engine Wash Frequency on GT Degradation in Natural Gas Compressor Stations, ASME Turbo Expo (2013) 